\title{
Theoretical Perspectives of Spin Dynamics in Solid-State Nuclear Magnetic Resonance and Physics
}

\author{
Eugene Stephane Mananga ${ }^{1,2,3}$ \\ ${ }^{1}$ The Graduate Center, The City University of New York, New York, USA \\ ${ }^{2}$ Department of Applied Physics, New York University, New York, USA \\ ${ }^{3}$ Department of Engineering, Physics, and Technology, BCC, The City University of New York, New York, USA \\ Email: esm041@mail.harvard.edu, emananga@gradcenter.cuny.edu
}

How to cite this paper: Mananga, E.S. (2018) Theoretical Perspectives of Spin Dynamics in Solid-State Nuclear Magnetic Resonance and Physics. Journal of Modern Physics, 9, 1645-1659. https://doi.org/10.4236/jmp.2018.98103

Received: May 23, 2018

Accepted: July 23, 2018

Published: July 26, 2018

Copyright $\odot 2018$ by author and Scientific Research Publishing Inc. This work is licensed under the Creative Commons Attribution International License (CC BY 4.0).

http://creativecommons.org/licenses/by/4.0/ (c) (i) Open Access

\begin{abstract}
Since the first demonstrations of nuclear magnetic resonance (NMR) in condensed matter in 1946, the field of NMR has yielded a continuous flow of conceptual advances and methodological innovations that continues today. Much progress has been made in the utilization of solid-state NMR to illuminate molecular structure and dynamics in systems not controllable by any other way. NMR deals with time-dependent perturbations of nuclear spin systems and solving the time-dependent Schrodinger equation is a central problem in quantum physics in general and solid-state NMR in particular. This theoretical perspective outlines the methods used to treat theoretical problems in solid-state NMR as well as the recent theoretical development of spin dynamics in NMR and physics. The purpose of this review is to unravel the versatility of theories in solid-state NMR and to present the recent theoretical developments of spin dynamics.
\end{abstract}

\section{Keywords}

Solid-state NMR, Hamiltonian Theory, Floquet Theory, Floquet-Magnus Expansion, Fer Expansion

\section{Introduction}

As front-line theories to control spin dynamics in solid-state nuclear magnetic resonance, the average Hamiltonian theory (AHT) and Floquet theory (FLT) have assumed great prominence and influence since the development of multiple pulse sequences and the inception of magic-angle spinning (MAS) methods in 
the 1960s [1] [2] [3] [4] [5]. The first endeavor in NMR to tackle with time-dependent Hamiltonians was built on the Magnus expansion that generated in AHT [6]-[12]. The AHT formalism describes how periodic pulse sequences can be used to control or transform the symmetry of selected interactions in coupled, many-spin systems, enabling the creation of effective Hamiltonians with fascinating and useful properties. From its natural formulation, this is the most widely used approach which has been applied to almost every kind of situation, sometimes abusively [13] [14] [15] [16] [17].

Floquet theory dissimilar to AHT, is not restricted to stroboscopic observation, yield a more universal approach for the description of the full time dependence of the response of a periodically time-dependent system [18] [19] [20] [21] [22] [23]. Methods developed over the past decade have enabled us to make a significant progress in the area of solid-state NMR by introducing an alternative expansion scheme called Floquet-Magnus expansion (FME) used to solve the time-dependent Schrodinger equation which is a central problem in quantum physics in general and solid-state NMR in particular [9] [11] [24]. The FME establish the connection between the ME and the Floquet theory, and provides a new version of the ME well suited for the Floquet theory for linear ordinary differential equations with periodic coefficients [9] [11] [24] [25] [26] [27]. We have proved that the ME is a particular case of the FME which yields new aspects not present in $\mathrm{ME}$ and Floquet theory such as recursive expansion scheme in Hilbert space that can facilitate the implementation of new or improvement of existing pulse sequences [24] [28]. In the same vein, Madhu and Kurur have recently introduced the Fer expansion (FE) in Solid-State NMR [29] [30]. The Fer expansion was formulated by Fer and later revised by Fer [29], Klarsfeld and Oteo [31], Casas et al. [32], and Blanes et al. [33]. This expansion employs the form of a product of sub-propagators, which appears to be suitable for examination of time-dependence of the density matrix for each average Hamiltonian at different orders. Some papers which outline the comparison of both theories (FME and FE) in NMR and physics were recently published in the solid-state NMR, chemical physics, and physics [34] [35].

\section{In the Beginning}

Historical overview of the first observations of NMR: Normally, credit for NMR first observation should go to Rabi and co-workers in 1939 who used a beam of silver atoms [36]. The noticeable change in the fluxes of beams representing the different energy states of the nuclear magnetic moments was the detection of transitions. However, the term NMR has come to be used as a convention for experiments, which differ from those of Rabi. The experiments set by the convention in respect of NMR are those through the detection of the transitions with the energy absorbed from the RF field rather than through changes in the particle flux reaching a detector as in the beam experiments. Next, the term NMR is commonly reserved for phenomena occurring in bulk matter rather 
than in a beam of essentially non-interacting atoms. As a result of these two important conventions, the first observations of NMR are attributed to two independent groups: Purcell, Torrey and Pound, working on the east coast of America and Bloch, Hansen and Packard working on the west coast. They published their discoveries almost simultaneously in the same volume of Physical Review in 1946 [37] [38] [39]. The following is the list of Nobel Price laureates awarded in NMR:

- Otto Stern, USA: Nobel Prize in Physics 1943, "for his contribution to the development of molecular ray method and his discovery of the magnetic moment of the proton".

- Isidor I. Rabi, USA: Nobel Prize in Physics 1944, "for his resonance method for recording the magnetic properties of atomic nuclei".

- Felix Bloch, USA and Edward M. Purcell, USA: Nobel Prize in Physics 1952, "for their discovery of new methods for nuclear magnetic precision measurements and discoveries in connection therewith".

- Richard R. Ernst, Switzerland: Nobel Prize in Chemistry 1991, "for his contributions to the development of the methodology of high resolution nuclear magnetic resonance (NMR) spectroscopy".

- Kurt Wüthrich, Switzerland: Nobel Prize in Chemistry 2002, "for his development of nuclear magnetic resonance spectroscopy for determining the three-dimensional structure of biological macromolecules in solution".

- Paul C. Lauterbur, USA and Peter Mansfield, United Kingdom: Nobel Prize in Physiology or Medicine 2003, "for their discoveries concerning magnetic resonance imaging".

An important landmark to describe the effect of time-dependent interactions and the accompanying improvements was the introduction of average Hamiltonian theory in solid-state NMR. Since its formal inception in 1968 by John Waugh, the average Hamiltonian theory has become the main tool to study the dynamics of spin systems subject to an RF perturbation and the most popular theoretical method in NMR. Its popularity stems from its excellently simple conceptual structure and computational elegance. AHT is a mathematical formalism that allows us to analyze how pulse sequences affect internal spin interactions. The rise of AHT in solid-state NMR began with the time-reversal experiments in dipolar-coupled spin systems [40]. Rhim and co-workers applieda suitable sequence of strong rf fields to a system of dipolar-coupled nuclear spins which was made to behave as though the sign of the dipolar Hamiltonian had been reversed. The system then appears to develop backward in time, and states of non-equilibrium magnetization can be recovered in systems which would superficially appear to have decayed to equilibrium. This behavior is consistent with dynamical and the rmodynamical principles, but shows that the spin-temperature hypothesis must be employed with caution [41] [42] [43]. The AHT is the most commonly used method to treat theoretical problems in solid-state NMR and have been used sometimes casually [17] [44]. As shown in Figure 1, the AHT method explains the average motion of the spin system, the effects of 
multiple-pulse sequences, and the effects of a time-dependent perturbation applied to the system.

The basic understanding of AHT involves considering a time dependent Hamiltonian $H(t)$ governing the spin system evolution, and describing the effective evolution by an average Hamiltonian $\bar{H}$ within a periodic time $(T)$. This is satisfied only if $H(t)$ is periodic $(T)$ and the observation is stroboscopic and synchronized with period $(T)$. Two major expansions (Baker-CambellHausdorff and Magnus) and an exact computation including the diagonalization of the time evolution operator defined the average Hamiltonian. This technique has been widely used in the NMR literature in the development of multiple pulse sequences and in the context of both decoupling and recoupling experiments. AHT is especially convenient in the derivation and analysis of pulse sequences that incorporate a block of $\mathrm{rf}$ irradiation that is repeated many times. The AHT set the stage for stroboscopic manipulations of spins and spin interactions by radio-frequency pulses and also explains how periodic pulses can be used to transform the symmetry of selected interactions in coupled, many-spin systems considering the average or effective Hamiltonian of the RF pulse train [21]. Today, AHT finds itself under increasing pressure of complicated experiments and to adapt to ever more challenging problems. If we are not mindful, under these pressures may submerge the introduction of Floquet theory [18] [20], Floquet-Magnus expansion [24], and Fer expansion in solid-state NMR [30].

\section{The Birth of Floquet Theory and Its Introduction to Solid-State NMR}

In 1883, M. Gaston Floquet proved a remarkable theorem that asserts the existence of a periodic unitary transformation that maps a system of normal differential equations with periodic coefficients into a system of differential equations with constant coefficients [2]. A well-known example of such a procedure is the passage to a rotating reference frame (RRF) in the study of a system with dipole-dipole interactions in a constant magnetic field and in a circularly polarized magnetic field. The Floquet theorem allows writing the solution of the Liouville evolution equation. Unfortunately, besides for the example with a RRF, the

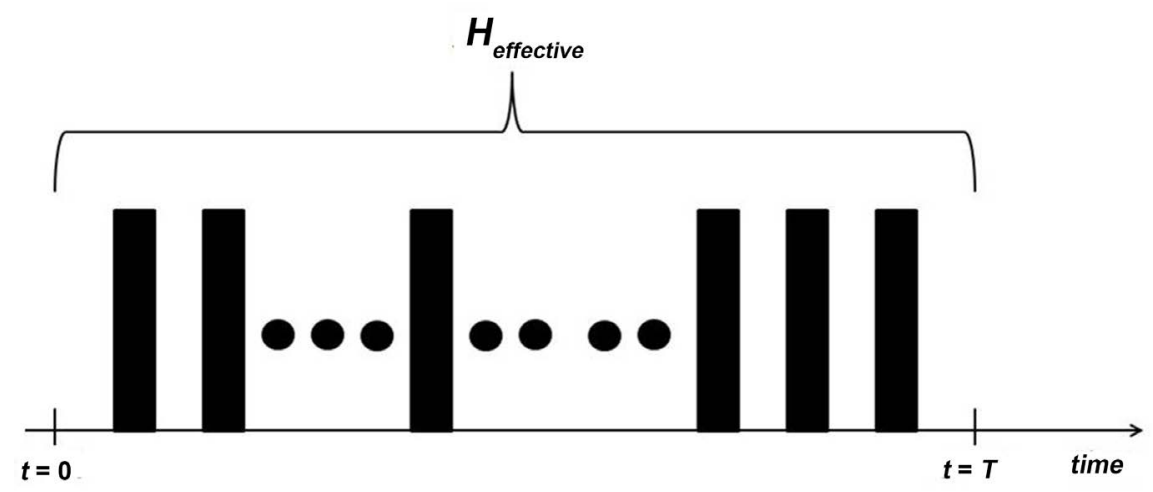

Figure 1. Basic Picture of average Hamiltonian theory. 
Floquet Hamiltonian for multi-spin systems cannot be calculated exactly, and approximate methods such as AHT should therefore be used [12]. The Floquet formalism approach for solving the Schrödinger equation with a Hamiltonian representing periodically time-dependent interactions was introduced to spectroscopy by Shirley in 1965 [3]. Shirley replaced the Hilbert space finite-dimensional time-dependent Hamiltonian by an infinite-dimensional time-independent Floquet Hamiltonian HF and showed how it can be used to obtain an exact solution to the LvN equation [19] [21] [22] [23] [24] [25]. Such an approach is often convenient, in particular, for describing solid-state NMR experiments, where the Hamiltonian becomes time dependent due to external manipulations such as MAS and/or periodic RF pulse trains. Shirley's Floquet formalism is also used in atomic and molecular spectroscopy methods [25]. The effective Hamiltonian derived using Floquet theory can be obtained in various ways. The most accurate way is to exactly diagonalise the Floquet Hamiltonian and transfer its diagonal form to the Hilbert space, taking into account the diagonalisation matrix. However, full diagonalisation can become very cumbersome and is rarely required. In most cases perturbation theory on HF can be applied, as done by Vega [20] [21]. Block diagonalisation methods, such as the van Vleck transformation, are extremely powerful in this case [17]. The resulting operator form of the effective Hamiltonian can then be used to design and evaluate the performance of NMR experiments. Solid-state NMR experiments are subjected to various time dependent perturbations of different frequencies, such as RF pulse schemes and MAS. We classify Hamiltonians into single mode, bimodal, and multimode depending on the number of distinct frequencies of perturbations to which the spin system is subjected [21]. Shirley's solution to the Schrödinger equation is also valid when the Hamiltonian is modulated by more than one periodic process [3]. Thus, Floquet theory provides a general description of many NMR experiments without placing any assumptions on the time scales of the perturbations.

\section{The Birth of Floquet-Magnus Expansion and Its Introduction to Solid-State NMR}

The Floquet-Magnus expansion was developed nearly a decade and half ago by Casas, Oteo, and Ros [11]. This approach is a new version of Magnus expansion well suited for Floquet theory of linear ordinary differential equations with periodic coefficients. Recently, the FME has been employed frequently for the treatment of quantum Floquet systems which open new possibilities to control quantum systems under periodic driving such as in quantum transport and quantum topological phases. The FME is a useful tool to treat a periodically driven system when the period $\mathrm{T}$ of the driving is very small. This approach is practically useful for the high-frequency driving, in which the higher-order contribution is not relevant to dynamics at short time scale. However, in the case of finite frequencies, the problem is more complicated since, in general, the FME is not convergent series expansion in the thermodynamic limit. More discussions on 
the physical meaning of the FME can be found in the literature. The connection between the Magnus expansion and the Floquet theory was established in the FME [24]. The FME approach is an alternative approach recently developed by Casas and co-workers to solve time-dependent linear differential equation which is a central problem in quantum physics in general and solid-state nuclear magnetic resonance (SSNMR) in particular [24]. The authors Casas, Oteo, and Ros build up a recursive scheme to obtain the terms in the new expansion and give an explicit sufficient condition for its convergence. The method and formulae were applied to an illustrative example from nuclear magnetic resonance, quantum mechanics, and physics [11]. When making the connection between the time-ordered products and the Magnus expansion, Oteo and Ros mentioned that the initial value problem originated from the linear homogeneous ordinary differential equation (LHODE) of first order [45]. The LHODE plays a pervasive role in many branches of mathematics, physics, and engineering with a wide range of different mathematical and physical meanings for the variable involved. Theoretical problems in solid-state NMR are widely treated with the average Hamiltonian theory and the Floquet theory. A variety of magnetic resonance phenomena has been described using both theories (AHT and FLT). These theories have been successful for designing sophisticated pulse sequences and understanding of different experiments. For instance, recently, the AHT has been used to develop a set of selection rules based on the symmetry of the internal interactions and Euler angles in order to simplify the design of NMR multiple-pulse sequences in the presence of sample rotation. This allows the development of recoupling and decoupling sequences as well as many other experiments. The selection rules reveal which types of interactions can be recoupled by a sequence with a given symmetry. Similarly, the FLT has been used to describe multiphoton effects in NMR, electron paramagnetic resonance, and nuclear quadrupole resonance. The fusion of AHT and FLT is generalized by the Floquet-Magnus expansion [1] [9] [24] [46] [47] [48] [49] [50]. The FME has been recently applied to various problems in solid-state NMR and Physics [51] [52] [53] [54] [55].

\section{The Birth of Fer Expansion and Its Introduction to Solid-State NMR}

The intuitive origins of Fer expansion date in the seminal 1958 Fer paper [29]. The FE approach is based on a factorization of the evolution operator as an infinite product of exponentials of Lie operators and thus exactly preserves the Poincaré integral invariants. This approach is an alternative expansion method to solving time-dependent linear differential equations. As already mentioned above, the FE was formulated by Fer and later revisited by Fer, Klarsfeld and Oteo, Casas et al., and Blanes et al. [29] [30] [31] [32] [33]. These authors illustrated some of the salient features of Fer expansion, and applied the method to simple cases such as a driven Harmonic oscillator, two-level system, and a gene- 
ralized simple harmonic oscillator. The FE approach was introduced recently to the NMR community by Madhu and Kurur via the effect of Bloch-Siegert shift and heteronuclear dipolar decoupling [30]. Unlike in Magnus expansion where an evaluation of nested commutators and their integrals are required to obtain the correction terms of a Hamiltonian, in Fer expansion only an evaluation of nested commutators is required [30]. As discussed in the next section, the convergence of Fer expansion is much faster than that of Magnus expansion, which lead to the calculation of the infinite number of commutators to be simple in most expereiments [30]. Indeed, from the point of view of physical applications, the Magnus expansion has been extensively used in a variety of issues, while the Fer expansion has been either ignored or misquoted until recently [35]. Both approaches are by no means equivalent, since, in general, the exponential operators do not commute with each other [25] [35]. The FE approach is still in its infancy in solid-state NMR and can be considered to be complimentary to the Magnus expansion (AHT) [34]. While the efficiency of Fer expansion seems obvious, more work is still required to allow the scheme to overcome difficulties such as cases involving non-periodic and non-cyclic cases [30].

\section{Convergence}

Setting an infinite sequence $\cdots\left(u_{1}, u_{2}, u_{3}, \cdots\right)$, the $n^{\text {th }}$ partial sum $\sigma_{n}$ is the sum of the first $n$ terms of the sequence,

$$
\sigma_{n}=\sum_{l=1}^{n} u_{l}
$$

A series is convergent if the sequence of its partial sums $\left\{\sigma_{1}, \sigma_{2}, \sigma_{3}, \cdots\right\}$ become closer and closer to a given number when the number of their terms increases. Mathematically speaking, a series converges, if there exists a number $p$ such that for any arbitrarily small positive number $\xi$ there is a large integer $N$ such that for all $n \geq N$

$$
\left|\sigma_{n}-p\right| \leq \xi
$$

If the series is convergent, the unique number $p$ is called the sum of the series. The Magnus and some of its equivalent such as Fer expansions have been applied to a wide range of problems in time-dependent quantum mechanics. Exponential time-dependent perturbation theories such as the Magnus expansion or Fer expansion, have proven useful in the treatment of a variety of problems in non-relativistic quantum dynamics. Until in the 1980's, very little was known about the convergence of exponential perturbation theory. In the original version, Magnus stated its convergence criterion in terms of the eigenvalues of the exponent itself. However, several groups have reported that application of the Magnus expansion in the Schrodinger representation to some problems of spectroscopy interest gave results which were less adequate [56]-[70]. Convergence of the Magnus expansion has also come into question in different applications. In general, the Magnus series does not converge unless the Hamiltonian is 
small in a suitable sense. Magnus expansion permits significantly simplifying the analysis of the behavior of spin systems in periodic external fields. However, divergence of the Magnus expansion guides to inconsistencies in the spin dynamics of solid-state NMR [8] [56]-[61]. Indeed, the convergence of the Magnus expansion is generally discussed in terms of a radius of convergence $r_{c}$. Several results on the radius of convergence $r_{c}$ in terms of the $H$ amiltonian have been obtained in the literature. Pechukas and Light and Karasev and Mosolova obtained a radius $r_{c}=\log 2=0.693 \cdots$ [62] [63], whereas Chacon and Fomenko [64] got a radius $r_{c}=0.577$. Blanes et al. obtained the improved bound radius of $r_{c}=1.086$ [65]. Recently, a new method was developed to enlarge the largest domain of convergence of the Magnus expansion $\left(r_{c}=1.086 \cdots\right)$ previously obtained [66]. An analytic estimate of the new domain of convergence found was almost twice the preceding one $\left(r_{c}=2\right)$ and this new analytic bound was in agreement with the numerical estimate of the convergence radius such as no accuracy was lost in the bound [67]. Therefore, there are more than three different convergence estimated in the literature of Magnus expansion. These convergence estimates are given with their respective proofs in the references therein. The latest improved bound $r_{c}=\pi$ was derived by Moan but in the context of the conventional Magnus expansion for real matrices [68]. This important results was then generalized to matrices in the Hilbert space (thus for complex matrices) by Casas [69]. A new version of Magnus expansion was reported recently by Butcher et al. [70]. The new scheme grows on trees and forests to reorder the terms of Magnus expansion for more efficient computation. While this scheme did not provide any substantial new result to the convergence of the ME, it provides a new mean to compute Magnus expansion to the desired order. This ME-type formalism has been developed in a more abstract setting of dendriform algebras. This form shows that the reordering of the terms in Magnus' expansion may be represented graphically using trees and forests, which may be collected into groups according to the order in time for which the solution is valid.

Recoupling schemes have all been extensively treated with Floquet theory inconjunction with the Van Vleck Transformation [17] [21] [23] [71]. The Floquet theory approach has also been used successfully to the study of decoupling of dipolar interactions. The discussion of the convergence of the Floquet theory was presented by Maricq [18]. Maricq first shows the convergence of the effective Hamiltonian in the mathematical sense. Next he elucidated by example, the conditions which must be satisfied in order to truncate the series for $\mathrm{P}(\mathrm{t})$ and $\mathrm{H}_{\mathrm{F}}$ after the first few terms. The appropriateness of the FME and FE are well related to the problem of convergence. This problem has played a pivotal role in the field of solid-state NMR and spin dynamics [8] [12] [25] [35]. FME and FE are divergent approaches and the physical nature of their divergences is discussed in the following paragraphs. The authors Casas, Oteo, and Ros investigated a sufficient condition for the absolute convergence of the FME in ref. [11], Blanes and co-workers studied succinctly the convergence of the Fer expansion by looking for conditions on the time dependent Hamiltonian [33]. The authors derived a 
convergent radius of the Fer expansion to be 0.8604065 and point out that, additional properties of the time dependent Hamiltonian allow an improvement of the result to extend the range of the radius originally given by Fer who used a slightly different argument [29]. In a similar vein, Zanna showed that a similar result holds for the symmetric Fer expansion by proving that the symmetric Fer expansion converges uniformly in the periodic interval [72].

This calculated radius, $\xi=0.60275$, of the convergence of the symmetric Fer expansion by Zanna is smaller than the calculated radius for the classical Fer expansion [72], $\xi=0.8604065$, by Blanes [33]. However, the bounds initiated by Zanna are not optimal and could be improved [72]. An important point of the FME approach is that the rate of convergence of the FME is faster than the Fer expansion in the sense that, for a prescribed precision, one needs more $F_{k}^{\prime} s$ (for Fer expansion) than $\Lambda_{k}^{\prime} s$ (for Floquet-Magnus expansion) even if from the computational point of view, the Fer expansion could require more work than the FME. The convergence of these approaches is extensively discussed in the literature [8] [11] [18] [24] [25] [35] [58] [59] [60] [65] [72].

\section{Applications of FME and FE in Physics}

Using the FME and FE approaches, many problems can be attacked in other fields of physics beyond the scope of NMR. It is important to remember that these considered methods have recently found new major areas of applications such as topological materials [73]. However, researchers dealing with these new applications are not usually acquainted with the achievements of the magnetic resonance theory, where those methods were developed more than thirty years ago [74] [75]. Researchers repeat the same mistakes that were made when the methods of spin dynamics and thermodynamics were developed in the past. Even though the FME is a divergent approach in general, its finite truncation can give useful information such as on the transient dynamics in periodically driven many-body quantum systems [73] [74]. Currently, the use of FME to obtain the effective Hamiltonians for periodically driven systems is a hot topic in the investigation of dynamics of classical and quantum systems. Recently, Kuwahara and co-workers showed that the finite truncation of the FME can give useful information on the transient dynamics [85]. The authors gave a rigorous relationship between the FME and general properties of transient quantum dynamics. New avenues of exploring FME and FE can also be extended to other areas of physics such as particles and high energy physics [25] [35]. These two approaches (FME and FE) can be used to solve problems in quantum field theory (QFT) and high energy physics, in particular problems similar to the one solved or fail to be solved by ME. For instance,

1) the ME has been used as an alternative to conventional perturbation theory for quantum fields to graph rules for functions of the time-evolution operator where normal products and Wick theorem were used. This was useful in the treatment of infrared divergences for some quantum electrodynamics process 
such as the scattering of an electron on an external potential or the bremsstrahlung of one hard photon [76] [77]. I believe that effective method of approaching this problem demands more inspection where FME and FE can play a major role;

2) an extension of the ME has been applied to the context of Connes-Kreimer's Hopf algebra approach to perturbative renormalization of quantum field theory showing that the generalized MEallows to solve the Bogoliubov-Atkinson recursion [78] [79] [80]. The FME and FE can also be applied in this context;

3) in the field of high energy physics, ME has also found applications such as to heavy ion collisions. ME is applied in collision problems when the use of unitary approximation scheme is necessary such as the unitary of the time evolution operator imposing some bound on the experimentally observable cross sections [80] [81]. FME and FE can also be used in this context as an intuitive method for simplifying calculations;

4) the problem in neutron oscillations which is closely related to solar neutrino problem. As neutrinos with different masses propagate with different velocities, the mixing allows for flavor conversion corresponding to neutrinos oscillations [82] [83] [84]. Fer's factorization as a symplectic integrator can, in principle, enter in the solution of the evolution operator in one basis.

The introduction of FME and FE as theoretical approaches to control the spin dynamics in the field of nuclear magnetic resonance are new exploratory and developmental researches which is a significant addition to the existing theoretical framework of AHT and FT. QFT is the basic mathematical language used to describe and analyze the physics of elementary particles. The theory by itself is an abstract representation for constructing quantum mechanics models of subatomic particles in particle physics and quasiparticles in condensed matter physics. The application of the FME and FE approaches as intuitive approaches in simplifying calculations to solve some specifics problems in the field of high energy physics and QFT such as those outlined in the above paragraph is of major interest. It is worth noting that, the FME has the advantage of having the unitary character of the evolution operator which is preserved at all orders of approximation while the $\mathrm{FE}$ has an advantage over the ME that only an evaluation of nested commutators is required in the calculation of the Hamiltonian [25] [35].

\section{Conclusion}

To summarize, our descriptions for all four theories suggest that the Fer expansion is advantageous over the other three theories (AHT, FLT, and FME) in calculation of higher-order corrections. As explained above, while the AHT and FLT are common in solid-state NMR, both the FME and Fer expansion are relatively newcomer although the mathematical formalism has been known for several decades [85]. One of the most salient features of the Fer expansion is that 
the first fifth-order average Hamitonians are sufficient to control an NMR experiment, and they are included in the first correction of the Fer expansion [86].

\section{Acknowledgements}

The author thank the CUNY Office Assistant Oana Teodorescu for reading and for editing the manuscript. He acknowledges the support from the CUNY GRANT CCRG\# 1517 and the CUNY RESEARCH SCHOLAR PROGRAM2017-2018. He also acknowledges the mentee's student Francesca Serrano for helping in editing the manuscript. The contents of this paper are solely the responsibility of the author and do not represent the official views of the NIH.

\section{Conflict of Interest}

The author declares that there is no conflict of interest regarding the publication of this paper.

\section{References}

[1] Haeberlen, U. and Waugh, J.S. (1968) Physical Review, 175, 453-467. https://doi.org/10.1103/PhysRev.175.453

[2] Floquet, M.G. (1883) Annales Scientifiques de PEcole Normale Superieure, 12, 47-88. https://doi.org/10.24033/asens.220

[3] Shirley, J.H. (1965) Physical Review B, 138, 979-987. https://doi.org/10.1103/PhysRev.138.B979

[4] Andrew, E.R., Bradbury, A. and Eades, R.G. (1958) Nature, 182, 1659. https://doi.org/10.1038/1821659a0

[5] Lowe, I.J. (1959) Physical Review Letters, 2, 285-287. https://doi.org/10.1103/PhysRevLett.2.285

[6] Evans, W. (1968) Annals of Physics, 48, 72-93. https://doi.org/10.1016/0003-4916(68)90270-4

[7] Haeberlen, U. (1976) High Resolution NMR in Solids. Advances in Magnetic Resonance, Supplement 1, Academic Press, New York.

[8] Feldman, E.B. (1984) Physical Letters A, 104, 479-481. https://doi.org/10.1016/0375-9601(84)90027-6

[9] Blanes, S., Casas, F., Oteo, J.A. and Ros, J. (2009) Physics Reports, 470, 151-238. https://doi.org/10.1016/j.physrep.2008.11.001

[10] Magnus, W. (1954) Communications Pure Applied Mathematics, VII, 649-673. https://doi.org/10.1002/cpa.3160070404

[11] Casas, F., Oteo, J.A. and Ros, J. (2001) Journal of Physics A: Mathematical and General, 34, 3379-3388. https://doi.org/10.1088/0305-4470/34/16/305

[12] Kuznetsova, E.I., Feldman, E.B. and Feldman, D.E. (2016) Physics Uspekhi, 59, 577-582. https://doi.org/10.3367/UFNe.2016.02.037753

[13] Vandersypen, L.M.K. and Chuang, I.L. (2004) Review of Modern Physics, 76, 1037-1069. https://doi.org/10.1103/RevModPhys.76.1037

[14] Mananga, E.S., Rumala, Y.S. and Boutis, G.S. (2006) Journal of Magnetic Resonance, 181, 296-303. https://doi.org/10.1016/j.jmr.2006.05.015

[15] Mananga, E.S., Roopchand, R., Rumala, Y.S. and Boutis, G.S. (2007) Journal of 
Magnetic Resonance, 185, 28-37. https://doi.org/10.1016/j.jmr.2006.10.016

[16] Mananga, E.S., Hsu, C.D., Ishmael Islam, S.T. and Boutis, G.S. (2008) Journal of Magnetic Resonance, 193, 10-22. https://doi.org/10.1016/j.jmr.2008.03.014

[17] Goldman, M., Grandinetti, P.J., Llor, A., Olejniczak, Z., Sachleben, J.R. and Zwanziger, J.W. (1992) Journal of Chemical Physics, 97, 8947-8960. https://doi.org/10.1063/1.463321

[18] Maricq, M.M. (1982) Physical Review B, 25, 6622. https://doi.org/10.1103/PhysRevB.25.6622

[19] Levante, T.O., Baldus, M., Meier, B.H. and Ernst, R.R. (1995) Molecular Physics, 86, 1195-1212. https://doi.org/10.1080/00268979500102671

[20] Vega, S., Olejniczak, E.T. and Griffin, R.G. (1984) Journal of Chemical Physics, 80, 4832-4840. https://doi.org/10.1063/1.446504

[21] Leskes, M., Madhu, P.K. and Vega, S. (2010) Progress in Nuclear Magnetic Resonance Spectroscopy, 57, 345-380. https://doi.org/10.1016/j.pnmrs.2010.06.002

[22] Scholz, I., Meier, B.H. and Ernst, M. (2007) Journal of Chemical Physics, 127, Article ID: 204504. https://doi.org/10.1063/1.2800319

[23] Scholz, I., Van Beek, J.D. and Ernst, M. (2010) Solid State Nuclear Magnetic Resonance, 37, 39-59. https://doi.org/10.1016/j.ssnmr.2010.04.003

[24] Mananga, E.S. and Charpentier, T. (2011) Journal of Chemical Physics, 135, Article ID: 044109. https://doi.org/10.1063/1.3610943

[25] Mananga, E.S. and Charpentier, T. (2016) Physics Reports, 609, 1-49. https://doi.org/10.1016/j.physrep.2015.10.005

[26] Provotorov, B.N. and Fel'dman, E.B. (1980) Journal of Experimental and Theoretical Physics, 52, 1116-1122.

[27] Fel'dman, E.B., Hitrin, A.K. and Provotorov, B.N. (1983) Physics Letters A, 99, 114-116. https://doi.org/10.1016/0375-9601(83)90938-6

[28] Mananga, E.S., Reid, A.E. and Charpentier, T. (2012) Solid State Nuclear Magnetic Resonance, 41, 32-47. https://doi.org/10.1016/j.ssnmr.2011.11.004

[29] Fer, F. (1958) Bulletin de la Classe des Sciences, 44, 818-829.

[30] Madhu, P.K. and Kurur, N.D. (2006) Chemical Physics Letters, 418, 235-238. https://doi.org/10.1016/j.cplett.2005.10.134

[31] Klarsfeld, S. and Oteo, J.A. (1989) Journal of Physics A: Mathematical and General, 22, 2687-2694. https://doi.org/10.1088/0305-4470/22/14/019

[32] Casa, F., Oteo, J.A. and Ros, J. (1991) Journal of Physics A: Mathematical and General, 24, 4037-4046. https://doi.org/10.1088/0305-4470/24/17/020

[33] Blanes, S., Casas, F., Oteo, J.A. and Ros, J. (1998) Journal of Physics A: Mathematical and General, 31, 259-268. https://doi.org/10.1088/0305-4470/31/1/023

[34] Mananga, E.S. (2013) Solid State Nuclear Magnetic Resonance, 55-56, 54-62. https://doi.org/10.1016/j.ssnmr.2013.08.002

[35] Mananga, E.S. (2016) Physics Reports, 608, 1-41. https://doi.org/10.1016/j.physrep.2015.10.006

[36] Rabi, I.I., Millman, S., Kusch, P. and Zacharias, J.R. (1939) Physical Review, 55, 526-535. https://doi.org/10.1103/PhysRev.55.526

[37] Mananga, E.S. (2005) High-Pressure Nuclear Magnetic Resonance Studies of Fuel Cell Membranes. Ph.D. Thesis, The City University of New York, New York. 
[38] Purcell, E.M., Torrey, H.C. and Pound, R.V. (1946) Physical Review, 69, 37-38. https://doi.org/10.1103/PhysRev.69.37

[39] Bloch, F., Hansen, W.W. and Packard, M. (1946) Physical Review, 70, 474-485. https://doi.org/10.1103/PhysRev.70.474

[40] Rhim, W.K., Pines, A. and Waugh, J.S. (1971) Physical Review B, 3, 684-695. https://doi.org/10.1103/PhysRevB.3.684

[41] Abragam, A. and Goldman, M. (1982) Nuclear Magnetism: Order and Disorder. Clarendon, Oxford.

[42] Ivanov, Y.N., Provotorov, B.N. and Fel'dman, E.B. (1978) Journal of Experimental and Theoretical Physics Letters, 27, 153-156.

[43] Ivanov, Y.N., Provotorov, B.N. and Fel'dman, E.B. (1978) Journal of Experimental and Theoretical Physics, 48, 930-936.

[44] Mananga, E.S. and Hu, B. (2016) Journal of Physical Chemistry A, 120, 8657-8679. https://doi.org/10.1021/acs.jpca.6b06595

[45] Oteo, J.A. and Ros, J. (2000) Journal of Mathematics Physics, 41, 3268-3277. https://doi.org/10.1063/1.533304

[46] Tycko, R. (2008) Journal of Chemical Physics, 128, Article ID: 052101. https://doi.org/10.1063/1.2833958

[47] Ernst, R.R., Bodenhausen, G. and Wokaun, A. (1987) Principles of Nuclear Magnetic Resonance in One and Two Dimension. Clarendon, Oxford.

[48] Vega, S. and Pines, A. (1977) Journal of Chemical Physics, 66, 5624-5644. https://doi.org/10.1063/1.433884

[49] Zax, D.B., Goelmn, G., Abramovich, D. and Vega, S. (1990) Advances in Magnetic and Optical Resonance, 14, 219-240. https://doi.org/10.1016/B978-0-12-025514-6.50016-2

[50] Abragam, A. (1961) The Principles of Nuclear Magnetism. Clarendon, Oxford.

[51] Mananga, E.S. (2013) Solid State Nuclear Magnetic Resonance, 54, 1-7. https://doi.org/10.1016/j.ssnmr.2013.04.001

[52] Mananga, E.S. (2014) Journal of Modern Physics, 5, 145-148. https://doi.org/10.4236/jmp.2014.54024

[53] Mananga, E.S. and Reid, A.E. (2013) Molecular Physics, 111, 243-257. https://doi.org/10.1080/00268976.2012.718379

[54] Mananga, E.S., Moghaddasi, J., Sana, A., Akinmoladun, A. and Sadoqi, M. (2015) Journal of Nature and Science, 1, e109.

[55] Mananga, E.S. and Charpentier, T. (2015) Chemical Physics, 450-451, 83-90. https://doi.org/10.1016/j.chemphys.2015.02.006

[56] Buishvili, L.L., Kobakhidze, G.V. and Menabde, M.G. (1982) Journal of Experimental and Theoretical Physics, 56, 347-349.

[57] Fel'dman, E.B. (1984) Journal of Experimental and Theoretical Physics Letters, 39, 657-659.

[58] Salzman, W.R. (1987) Physical Review A, 36, 5074-5076. https://doi.org/10.1103/PhysRevA.36.5074

[59] Salzman, W.R. (1985) Journal of Chemical Physics, 82, 822-826. https://doi.org/10.1063/1.448508

[60] Salzman, W.R. (1986) Chemical Physics Letters, 124, 531-533. https://doi.org/10.1016/0009-2614(86)85070-9 
[61] Llor, A. (1992) Chemical Physics Letters, 199, 383-390. https://doi.org/10.1016/0009-2614(92)80136-Y

[62] Pechukas, P. and Light, J.C. (1966) Journal of Chemical Physics, 44, 3897-3912. https://doi.org/10.1063/1.1726550

[63] Karasev, M.V. and Mosolova, M.V. (1977) Theoretical Mathematics and Physics, 28, 721-730. https://doi.org/10.1007/BF01029029

[64] Chacon, R.V. and Fomenko, A.T. (1991) Advances in Mathematics, 88, 200-257. https://doi.org/10.1016/0001-8708(91)90008-U

[65] Mananga, E.S. (2018) Physica B: Condensed Matter Physics, 528, 47-59. https://doi.org/10.1016/j.physb.2017.10.087

[66] Moan, P.C. and Oteo, J.A. (2001) Journal of Mathematics Physics, 42, 501-508. https://doi.org/10.1063/1.1330198

[67] Blanes, S., Casas, F., Oteo, J.A. and Ros, J. (1998) Journal of Physics A: Mathematical and General, 31, 259-268. https://doi.org/10.1088/0305-4470/31/1/023

[68] Moan, P.C. and Niesen, J. (2008) Foundation of Computations Mathematics, 8, 291-301. https://doi.org/10.1007/s10208-007-9010-0

[69] Casas, F. (2007) Journal of Physics A: Mathematical and Theoretical, 40, 15001-15017. https://doi.org/10.1088/1751-8113/40/50/006

[70] Butcher, E.A., Sari, M., Bueler, E. and Carlson, T. (2009) Communications in Nonlinear Science and Numerical Simulation, 14, 4226-4245.

https://doi.org/10.1016/j.cnsns.2009.02.030

[71] Van Vleck, J.H. (1929) Physical Review, 33, 467-506. https://doi.org/10.1103/PhysRev.33.467

[72] Mananga, E.S. (2014) Journal of Modern Physics, 5, 458-463. https://doi.org/10.4236/jmp.2014.56055

[73] Mikama, T., Kitamura, S., Yasuda, K., Tsuji, N., Oka, T., et al. (2016) Physical Review $B, 93$, Article ID: 144307.

[74] Eckardt, A. and Anisimovas, E. (2015) New Journal of Physics, 17, Article ID: 093039. https://doi.org/10.1088/1367-2630/17/9/093039

[75] Kuwahara, T., Mori, T. and Saito, K. (2016) Annals of Physics, 367, 96-124. https://doi.org/10.1016/j.aop.2016.01.012

[76] Dahmen, H.D., Mannel, T. and Manakos, P. (1988) Physical Review D, Part Fields, 38, 1176-1182. https://doi.org/10.1103/PhysRevD.38.1176

[77] Dahmen, H.D., Manakos, P., Mannel, T. and Ohl, T. (1991) Zeitschrift fur Physik C Particles and Fields, 50, 75-84.

[78] Connes, A. and Kreimer, D. (2000) Communication in Mathematical Physics, 210, 249-273. https://doi.org/10.1007/s002200050779

[79] Connes, A. and Kreimer, D. (2001) Communication in Mathematical Physics, 216, 215-241. https://doi.org/10.1007/PL00005547

[80] Ebrahimi-Fard, K., Manchon, D. and Patras, F. (2008) Journal of Algebra, 320, 708-727. https://doi.org/10.1016/j.jalgebra.2007.12.013

[81] Ionescu, D.C. (1994) Physical Review A, 49, 3188-3195. https://doi.org/10.1103/PhysRevA.49.3188

[82] DOlivo, J.C. (1992) Physical Review D, 45, 924-930. https://doi.org/10.1103/PhysRevD.45.924 
[83] DOlivo, J.C. and Oteo, J.A. (1996) Physical Review D, 54, 1187-1193. https://doi.org/10.1103/PhysRevD.54.1187

[84] Supanitsky, A.D., DOlivo, J.C. and Medina Tanco, G. (2008) Physical Review D, 78, Article ID: 045024. https://doi.org/10.1103/PhysRevD.78.045024

[85] Kuwahara, T., Mori, T. and Saito, K. (2016) Annals of Physics, 367, 96-124. https://doi.org/10.1016/j.aop.2016.01.012

[86] Takegoshi, K., Miyazawa, N., Sharma, K. and Madhu, P.K. (2015) Journal of Chemical Physics, 142, Article ID: 134201. https://doi.org/10.1063/1.4916324 